\title{
Polyethylene Naphthalate Scintillator: A Novel Detector for the Dosimetry of Radioactive Ophthalmic Applicators
}

\author{
Dirk Flühs $^{a}$ Andrea Flühs $^{a}$ Melanie Ebenau ${ }^{a, b}$ Marion Eichmann ${ }^{b}$ \\ a Universitätsklinikum Essen, Essen, and ${ }^{\mathrm{b}}$ Technische Universität Dortmund, Dortmund, Germany
}

\section{Key Words}

Dosimetry · Ophthalmic plaque · Polyvinyltoluene

scintillators · Polyethylene naphthalate scintillators

\begin{abstract}
Background: Dosimetric measurements in small radiation fields with large gradients, such as eye plaque dosimetry with $\beta$ or low-energy photon emitters, require dosimetrically almost water-equivalent detectors with volumes of $<1 \mathrm{~mm}^{3}$ and linear responses over several orders of magnitude. Polyvinyltoluene-based scintillators fulfil these conditions. Hence, they are a standard for such applications. However, they show disadvantages with regard to certain material properties and their dosimetric behaviour towards low-energy photons. Purpose, Materials and Methods: Polyethylene naphthalate, recently recognized as a scintillator, offers chemical, physical and basic dosimetric properties superior to polyvinyltoluene. Its general applicability as a clinical dosimeter, however, has not been shown yet. To prove this applicability, extensive measurements at several clinical photon and electron radiation sources, ranging from ophthalmic plaques to a linear accelerator, were performed. Results: For all radiation qualities under investigation, covering a wide range of dose rates, a linearity of the detector response to the dose was shown. Conclusion:
\end{abstract}

Polyethylene naphthalate proved to be a suitable detector material for the dosimetry of ophthalmic plaques, including low-energy photon emitters and other small radiation fields. Due to superior properties, it has the potential to replace polyvinyltoluene as the standard scintillator for such applications.

(c) 2015 S. Karger AG, Basel

\section{Introduction}

Brachytherapy with radioactive ophthalmic applicators is an effective treatment modality for tumours of the eye $[1,2]$. These plaques emit either $\beta$ radiation or lowenergy photons, and the resulting radiation fields are characterized by steep dose fall-offs within a few millimetres. Hence, the dosimetry necessary for quality assurance and for the development of new plaques is still a challenge and requires experience and appropriate detector systems.

For dosimetric tasks in a hospital, water is usually the reference medium, and measurements are performed in water or in a water-equivalent phantom material. Thus, a detector material with a density and atomic composition which is as close as possible to that of water can be an advantageous choice, since it minimizes the disturbance of

\section{KARGER}

E-Mail karger@karger.com

www.karger.com/oop
(C) 2015 S. Karger AG, Basel

2296-4681/15/0021-0005\$39.50/0
Dirk Flühs

Universitätsklinikum Essen

Hufelandstrasse 55

DE-45147 Essen (Germany)

E-Mail dirk.fluehs@uni-due.de 
the radiation field during the measuring process. This property is useful especially under measuring situations with large dose gradients or without secondary electron equilibrium - a precondition required for the usage of cavity detectors, such as ionization chambers [3]. Examples of such special and often problematic measuring conditions are radiation fields of eye plaques, as mentioned before, and photon and electron radiation fields of a clinical linear accelerator (LINAC) with sizes of about $40 \mathrm{~mm}$ or less, for instance in case of retinoblastoma treatment. In such situations, detector volumes of $1 \mathrm{~mm}^{3}$ or less are necessary in order to provide a sufficient spatial resolution. Plastic scintillators fulfil such requirements and have, therefore, been used in a wide range of electron and photon radiation dosimetries for decades, with different measuring geometries [4-19]. Furthermore, they allow for a direct reading of the signal proportional to the dose rate over four orders of magnitude and can be used as scanning detectors in a 3-dimensional water phantom $[6,19-21]$. Unlike dosimetric measurements with radiochromic films or thermoluminescent dosimeters, the complete dose distribution of an ophthalmic plaque can be measured with a scintillation dosimeter within a single measuring procedure, i.e. one scan across the whole region of interest, without any re-calibration of the detector system. Due to these characteristics, dosimetry systems with plastic scintillators are a standard for eye plaque dosimetry today [6,19-23], in spite of the fact that they usually have to be manufactured by the users themselves, since no commercial supplier offers such systems due to the small market.

A scintillation dosimeter system typically consists of the scintillator itself, a suitable light-guiding tube for the transmission of the emitted scintillation light and a photomultiplier or a photodiode read out by an amperemeter for the determination of the light intensity. Cerenkov radiation, i.e. light generated by the (secondary) electrons within the light-guiding tube, causes a considerable contribution to the total signal strength and has to be taken into account in most measuring situations. In order to solve this problem, sophisticated solutions have been presented $[6,12,19,24-29]$.

The ultraviolet-emitting scintillator polyvinyltoluene (PVT) has been the most commonly used base material for scintillation dosimeters so far. Added wavelength shifters, such as POPOP and p-terphenyl, result in a light emission of the plastic scintillator within the visible spectrum. The widely used type BC-400, for instance, has an emission maximum at a wavelength of $423 \mathrm{~nm}$ in the blue range of light [30].

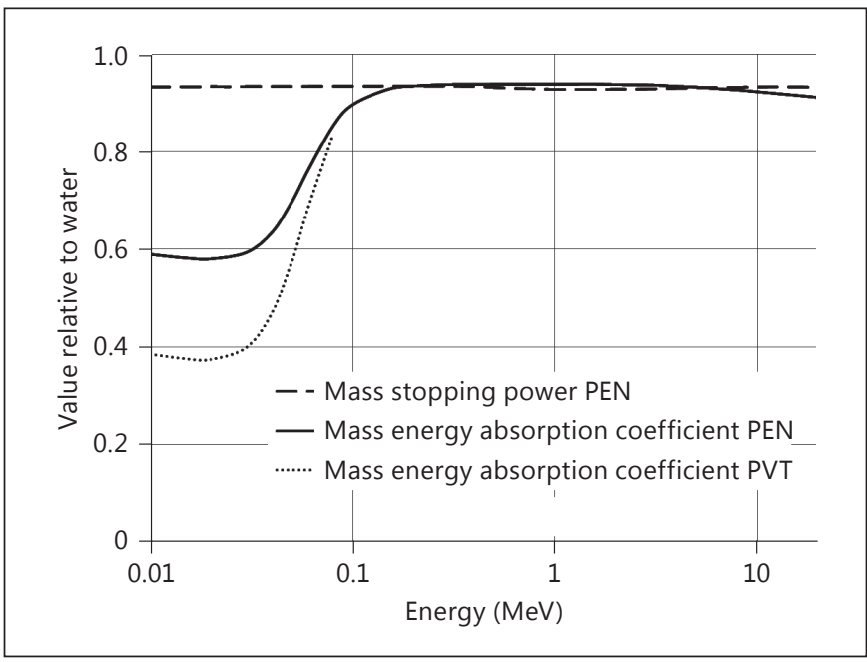

Fig. 1. Ratio of the mass energy absorption coefficient and mass stopping power of PEN and water. For comparison, the ratio of the mass energy absorption coefficient of PVT and water in the lowenergy range is given as well.

In contrast to PVT, polyethylene naphthalate (PEN) is a recently discovered scintillator that does not contain any wavelength shifter but directly emits blue light [31]. It is mechanically far more stable and heat resistant than PVT. Hence, we expected better and more easily obtainable results for manufacturing very small dosimeters from this material, instead of PVT, especially for the dosimetry of small radiation fields and ophthalmic applicators. An essential precondition for this usage, however, was the determination of the basic dosimetric properties of PEN and its general applicability as a dosimeter for clinical electron and photon beams. This applicability is demonstrated in the following.

\section{Materials and Methods}

PEN is a polyester with a density of about $1.35 \mathrm{~g} / \mathrm{cm}^{3}$. It is a thermoplastic with a melting point of about $270^{\circ} \mathrm{C}$ and improved chemical and hydrolytic resistance compared to PVT. The atomic composition is $\left[\mathrm{C}_{14} \mathrm{H}_{10} \mathrm{O}_{4}\right]_{\mathrm{n}}$. It was recognized as a scintillator with good light yield in 2011 [31]. Basic dosimetric properties of this material, with respect to the measurements of the absorbed dose to water, are described by the ratios of the mass energy absorption coefficient of PEN and water for photons, as already mentioned before, and the ratio of the mass stopping power for electrons, respectively. The necessary data can be obtained from NIST [32, 33]. The ratios between $10 \mathrm{keV}$ and $20 \mathrm{MeV}$ are shown in figure 1 . The ratio of the mass stopping powers remains constant within a few percent in the whole energy range of dosimetric interest. The ratio 
of the energy absorption coefficient shows the typical behaviour of many other plastics: an almost constant ratio above $150 \mathrm{keV}$, where the Compton effect is dominating, and again only slight variations in the energy range below $40 \mathrm{keV}$, with a dominating photo-electric effect. From $100 \mathrm{keV}$ down to $40 \mathrm{keV}$, there is a decrease of the ratio by a factor of 0.6. However, the decrease is smaller for PEN than for PVT, where the value goes down to 0.4 [33].

Our investigation program for the PEN scintillator consisted of three steps:

1 A comparison of the measuring values of the scintillator and an ionization chamber in a simple measuring setup for a ${ }^{192} \mathrm{Ir}$ afterloading source was performed as a first check of the linearity of the scintillator signal to the dose.

2 More detailed measurements were performed for photon and electron beams of a linear accelerator (LINAC). Sufficient field sizes allowed the usage of calibrated standard detectors for the dosimetric routine in the hospital, i.e. ionization chambers, as an accurate reference.

3 After these basic investigations, we finally measured the depth dose profiles of two ophthalmic plaques, one with the $\beta$ emitter ${ }^{106} \mathrm{Ru} /{ }^{106} \mathrm{Rh}$ (BEBIG type CCB, diameter $20.2 \mathrm{~mm}$ ) and the other with the low-energy photon emitter ${ }^{125} \mathrm{I}$, with a PEN scintillator. Since only applicators with ${ }^{106} \mathrm{Ru} /{ }^{106} \mathrm{Rh}$ or both ${ }^{125} \mathrm{I}$ and ${ }^{106} \mathrm{Ru} /{ }^{106} \mathrm{Rh}$ were available from our current clinical routine, the ${ }^{125}$ I plaque was especially constructed for these measurements. It was based on a CCB-shaped gold calotte with 8 seeds (BEBIG type IsoSeed ${ }^{\circledR}$ I25.S16), all of them positioned close to its centre. As a reference detector, we used a calibrated dosimetry system with a PVT scintillator, traceable to a secondary standard, provided by the German National Metrology Institute, Physikalisch-Technische Bundesanstalt.

For each of these three setups, we manufactured an appropriate detector. We first formed a cylinder with a thickness of $5 \mathrm{~mm}$ and a diameter of $25 \mathrm{~mm}$ from pure PEN granulate (Goodfellow $\mathrm{GmbH}$ ) that was heated in an oven to a temperature of $275^{\circ} \mathrm{C}$. From this cylinder, pieces of different sizes were cut out with a metal saw: a piece of $5 \times 5 \times 5 \mathrm{~mm}^{3}$ for the measurement of the ${ }^{192} \mathrm{Ir}$ source used for afterloading brachytherapy, a piece of $1 \times 1 \times$ $1 \mathrm{~mm}^{3}$ for the measurements of the ${ }^{106} \mathrm{Ru} /{ }^{106} \mathrm{Rh}$ and the ${ }^{125} \mathrm{I}$ radioactive eye applicator and a piece with a base area of $5 \times 4 \mathrm{~mm}^{2}$ and a thickness of $2 \mathrm{~mm}$ for measurements of photon and electron beams of the clinical LINAC. After cutting, the pieces were polished by means of a plastic polish. These procedures were performed without the problems typically connected with the manufacturing of PVT: softening due to frictional heating, surface damage due to mechanical or chemical influences and the emission of toxic substances, such as toluene.

A cyanoacrylate glue (Sekundenkleber, UHU GmbH) was used to connect the PEN pieces to PMMA light-guiding tubes (Cunz $\mathrm{GmbH}$ ) with a diameter of $1 \mathrm{~mm}$. The light-guiding tubes had a length of $0.5 \mathrm{~m}$ (brachytherapy measurements) and $4 \mathrm{~m}$ (LINAC measurements), respectively. The scintillators were then covered by a thin layer of white acrylic paint in order to increase the light gain and, subsequently, together with the adjacent part of the lightguiding tubes, by black acrylic paint and a black shrinking tube in order to achieve light tightness of the system (fig. 2). The lightguiding tubes were furnished with light-tight optical fibre connectors and connected to the photocathode of Hamamatsu R647-01 photomultiplier tubes. This photomultiplier, a 10-stage tube, showed an amplification factor of typically $10^{6}$ at a supply voltage

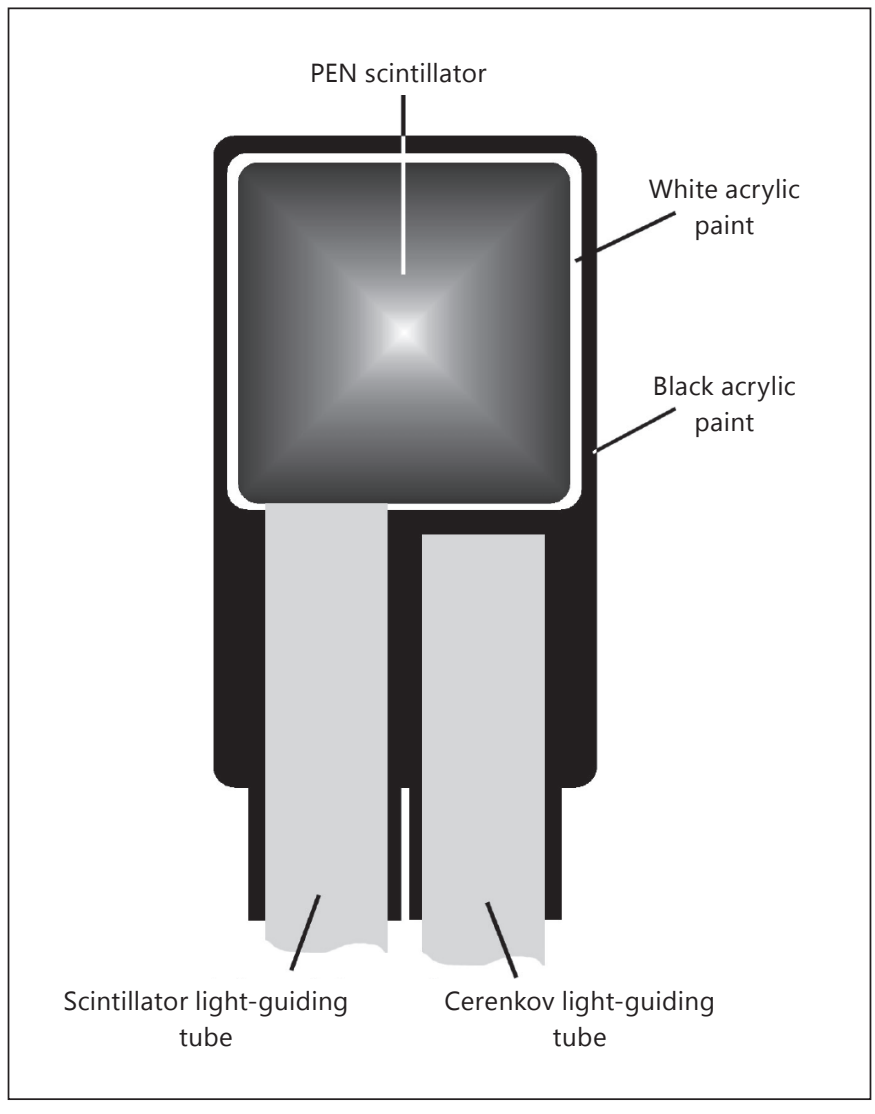

Fig. 2. Basic setup of a PEN scintillator with light-guiding tube for the transmission of the scintillation light (left) and second lightguiding tube for the determination of the Cerenkov light (right).

of $1 \mathrm{kV}$ in our measuring setup. In case of the LINAC measurements, the photomultiplier tubes were read out by two electrometers (PTW Unidos 10001, PTW Freiburg GmbH, Freiburg, Germany), in all other cases by a picoamperemeter (Keithley 486, Keithley Instruments Inc.). Before further processing, all measuring data were corrected for the dark current of the photomultiplier tubes.

For the initial measurement of the ${ }^{192}$ Ir source, the PEN scintillator and a PTW 31003 ionization chamber, as a reference, were mounted side by side behind a plastic build-up cap for a simultaneous measurement. The signal of the ionization chamber was measured by a PTW Unidos 10001. The full length of the lightguiding tube and the photomultiplier tube itself were almost completely shielded from the radiation field by 5 -cm-thick lead blocks, thus reducing the radiation strength to $<1 \%$ (fig. $3 \mathrm{c}$ ). Correction for the Cerenkov effect was therefore unnecessary. In order to vary the dose rate, the radiation source was automatically moved to desired measuring positions within its guiding tube.

In the case of the measurements of the LINAC (Varian Clinac $2100 \mathrm{C}$ ), the detectors were precisely positioned at different positions in a water phantom PTW 4322. A second light-guiding tube (Cerenkov channel), shielded from the scintillator light, was mounted parallel to the first one (scintillator channel) in order to 
Fig. 3. Setup for measurements of beams from a LINAC (a), of radioactive ophthalmic applicators (b) and of the ${ }^{192} \mathrm{Ir}$ source (c). Here, 'dosimeter' represents both a PEN scintillator and an ionization chamber.

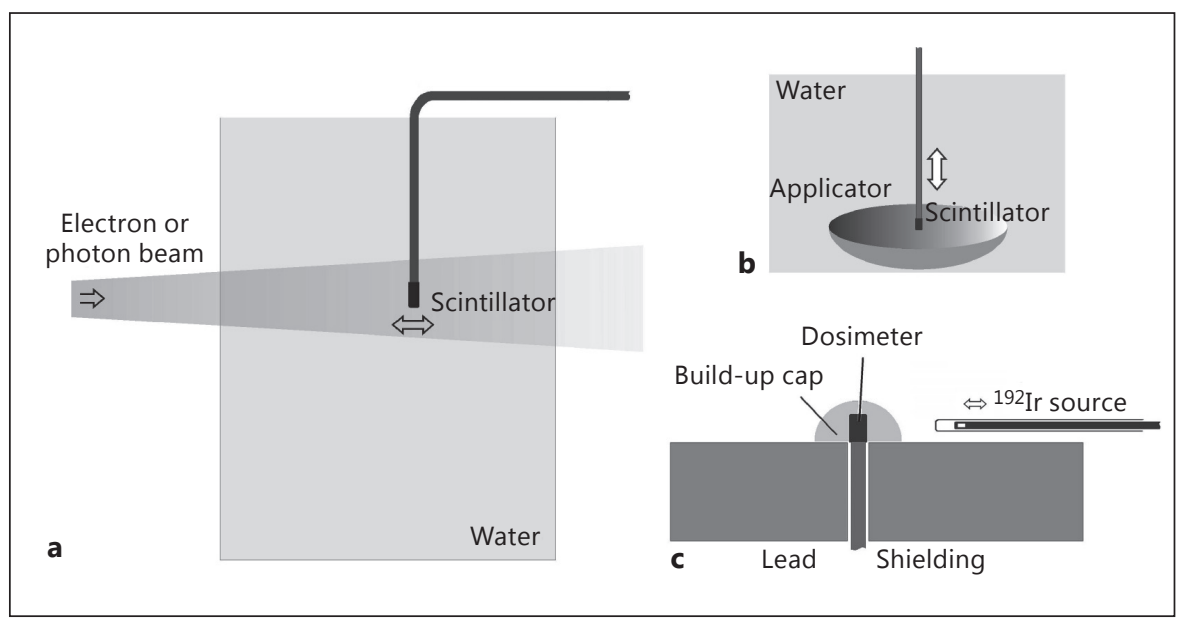

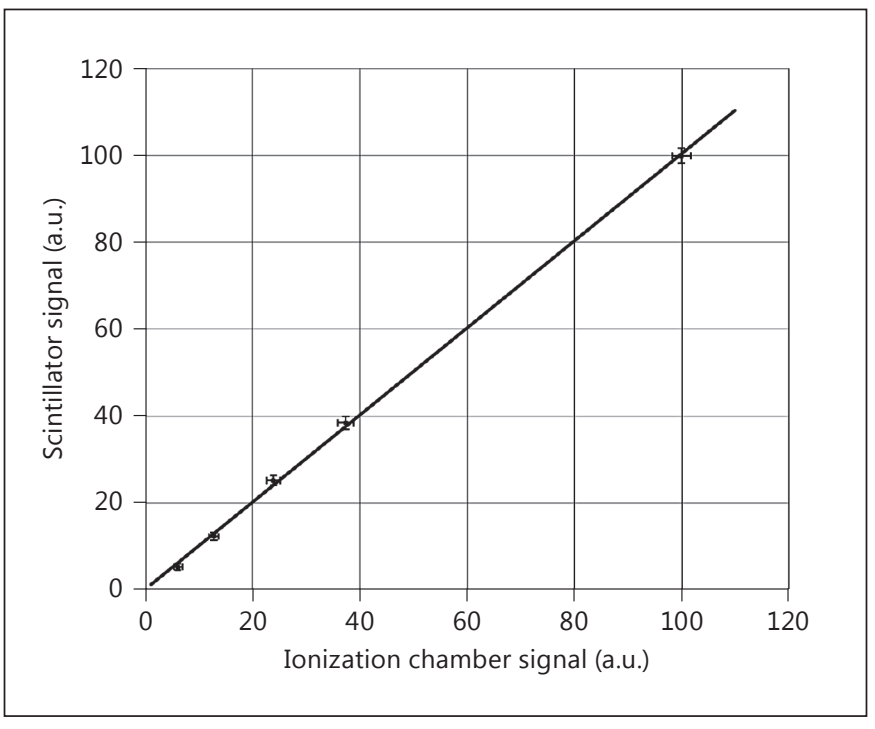

Fig. 4. Comparison between measurements (in arbitrary units; a.u.) of the ${ }^{192} \mathrm{Ir}$ source with a PEN scintillator and an ionization chamber. The linear correlation coefficient was $\mathrm{R}^{2}=0.9994$. The dose rate at the normalization point (100\%) was about $400 \mathrm{mGy} / \mathrm{min}$.

measure the amount of Cerenkov light generated in the light-guiding tube (fig. 3a). After relative calibration, the signal of the Cerenkov channel was subtracted from the total signal of the scintillator channel, thus enabling the determination of the pure scintillator signal which was compared to measurements with an ionization chamber PTW 31003 and electrometer PTW Unidos 10001. The measurements were not carried out simultaneously due to the reproducibility of the LINAC of $>0.5 \%$. The effective measuring point of the scintillator was set to its geometric centre, while for the ionization chamber, it was $1 / 2$ of the inner radius of the measuring volume towards focus (in accordance with DIN 6800-1 and DIN 6800-2 [34, 35]). The measurements were performed for beam energies of 6 and $15 \mathrm{MV}$ for photons, and 6 and $12 \mathrm{MeV}$ for electrons, at a pulse dose of $0.4-0.7 \mathrm{mGy}$ and a focus surface dis-

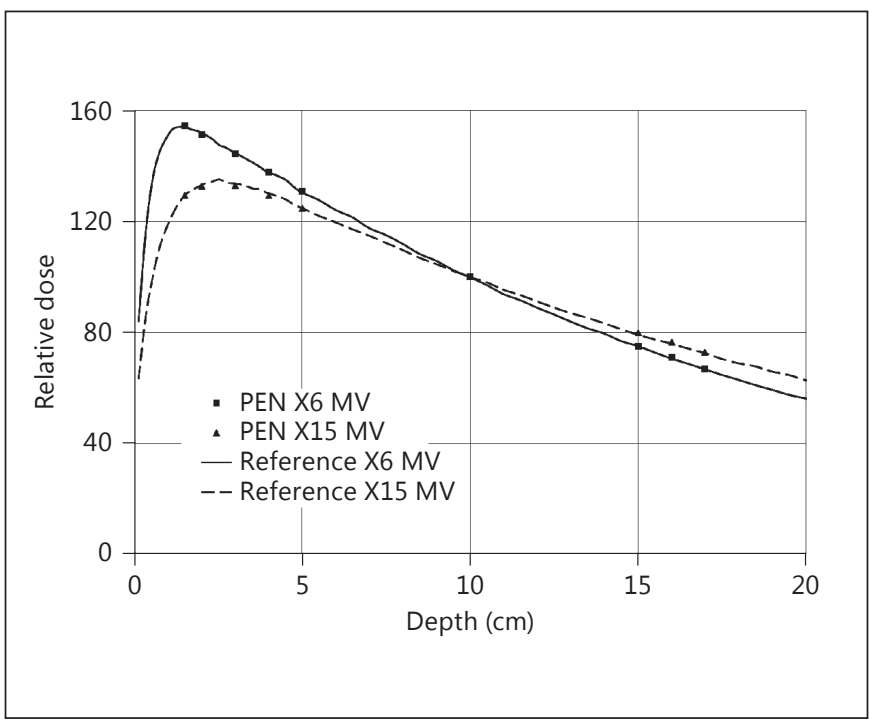

Fig. 5. Comparison between measurements of photon beams from a LINAC with a PEN scintillator and an ionization chamber. The dose rate at the normalization point at $10 \mathrm{~cm}$ was $1.6 \mathrm{~Gy} / \mathrm{min}$ for the 6-MV beam and $1.8 \mathrm{~Gy} / \mathrm{min}$ for the $15-\mathrm{MV}$ beam.

tance of $100 \mathrm{~cm}$. With $4-\mu \mathrm{s}$ mean width of a rectangular pulse, these values correspond to short-time dose rates of 92-175 Gy/s. The typical mean dose rates along the profiles for the chosen pulse repetition rates during the measurements were in the order of 10 $\mathrm{Gy} / \mathrm{min}$. The field sizes were $10 \times 10 \mathrm{~cm}^{2}$ for the photon beams and $5 \times 5 \mathrm{~cm}^{2}$ for the electron beams, and the detector was positioned at the field centre. Thus, secondary electron equilibrium for the photon beam was preserved. For the electron beam, the radiation field was sufficiently homogenous across the detector base area, with a field gradient only perpendicular to it.

For the final measurements of the eye applicators, the detector was automatically moved in a water phantom by a scanner along the central axis of the plaque (fig. 3b). No second light guide was used, however, and no correction for the Cerenkov light was per- 


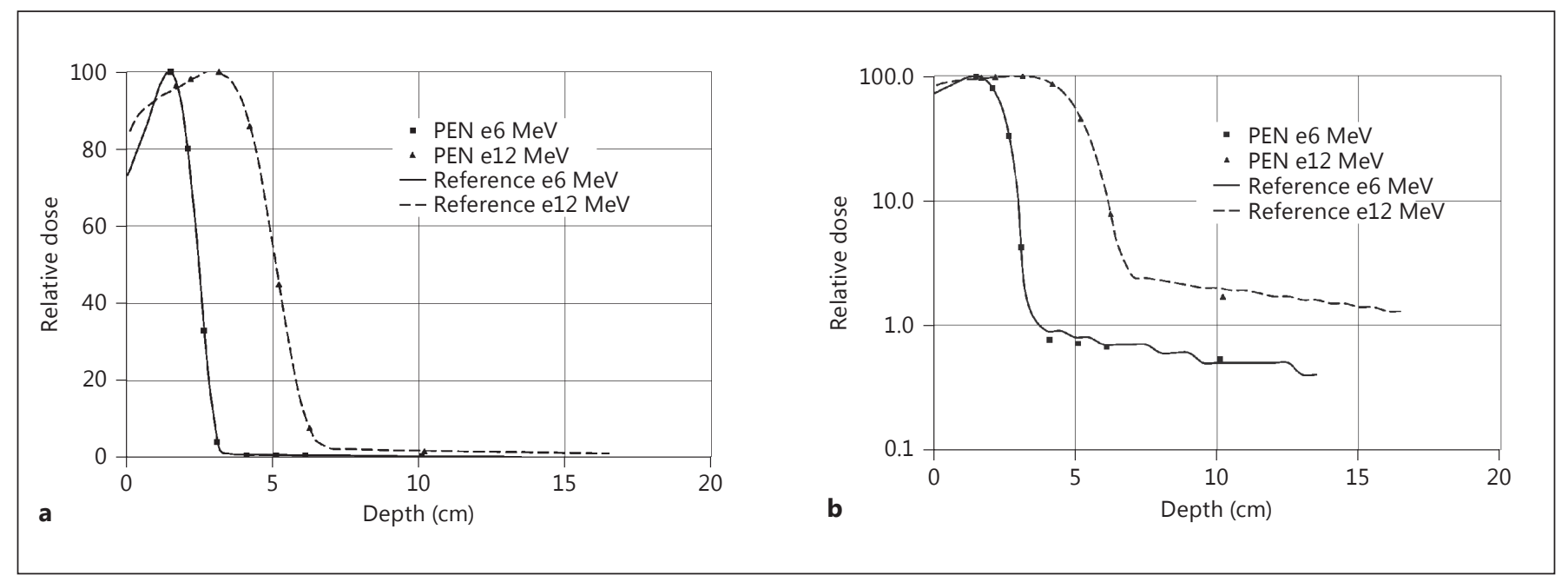

Fig. 6. Comparison between measurements of electron beams from a LINAC with a PEN scintillator and an ionization chamber, linear (a) and logarithmic (b) display. The dose rate at the maximum of the depth dose profiles was about $1.2 \mathrm{~Gy} / \mathrm{min}$.

formed either. This is unnecessary in case of ${ }^{125} \mathrm{I}$ due to the low photon energy below $35 \mathrm{keV}$. For an eye applicator containing ${ }^{106} \mathrm{Ru} /{ }^{106} \mathrm{Rh}$, a $\beta$ emitter with a maximal energy of $3.5 \mathrm{MeV}$, we expected the amount of Cerenkov light to be negligible within $\pm 5 \%$. This was shown for PVT scintillators of that size in previous measurements, under such measuring geometries [22], and the light output of PEN was expected to be similar to that of PVT [31] - a fact that was confirmed during our measurements. As a reference detector, we used a calibrated dosimeter based on a PVT scintillator, as mentioned above.

\section{Results}

For all radiation qualities under investigation, the obtained signals from PEN-based scintillators demonstrated a fine linear response to the dose rate, as measured by the reference dosimeters. This was proven valid even for the comparison of PEN and ionization chamber measurements of the ${ }^{192} \mathrm{Ir}$ afterloading source, in spite of the fact that this measurement was not performed in a suitable phantom. In this case, however, the chosen setup guaranteed the reproducibility of the measuring conditions (fig. 4). In the following, the uncertainties are given with a confidence interval of $k=1$.

For photon beams from the LINAC, the agreement of the depth dose profiles was within $1 \%$ for both energies (fig. 5). For electron beams from the LINAC, the initial increase and the steep dose fall-off of the depth dose profiles were demonstrated within $2 \%$ (fig. 6a). The small amount of bremsstrahlung background could also be determined, as figure $6 \mathrm{~b}$ shows, although the poorer measuring statistics were in the order of $\pm 10 \%$. It should be noted that the amount of light from the scintillator was so large that even with a value of $100 \mathrm{MU} / \mathrm{min}$ as the lowest pulse repetition rate of the LINAC, the supply voltage of the photomultipliers had to be reduced from 1,000 to 700 $\mathrm{V}$ in order to avoid an overflow of the electrometers in the highest measuring range.

Measurements of the low-energy photon field of a ${ }^{125} \mathrm{I}$ eye applicator, covering two orders of magnitude in dose rates from about 2-200 $\mathrm{mGy} / \mathrm{min}$, showed an excellent agreement within $2 \%$ to a measurement with a PVT dosimeter (fig. 7). For the ${ }^{106} \mathrm{Ru} /{ }^{106} \mathrm{Rh}$ eye plaque, the measuring values were in the expected agreement over nearly four orders of magnitude, from $100 \mathrm{mGy} / \mathrm{min}$ down to only 0.03 $\mathrm{mGy} / \mathrm{min}$ (fig. 8). Until a depth of $12 \mathrm{~mm}$, an agreement within $2 \%$ was observed as well. For clinically almost irrelevant depths larger than $12 \mathrm{~mm}$, the measuring statistics became significantly worse (up to $\pm 20 \%$ ), especially for the region beyond the maximal $\beta$ range of about $15 \mathrm{~mm}$. Nevertheless, the qualitative agreement between the results of both detectors in this region was acceptable, especially when taking into account the very small dose rate.

Under the same measuring conditions, we found the ratio of the absolute response values of the PEN and PVT scintillator to the radiation of low-energy photons ( 1.78 $\pm 0.06)$ to be almost twice as high as the ratio of the response values to $\beta$ radiation (1.01 \pm 0.03 ). 


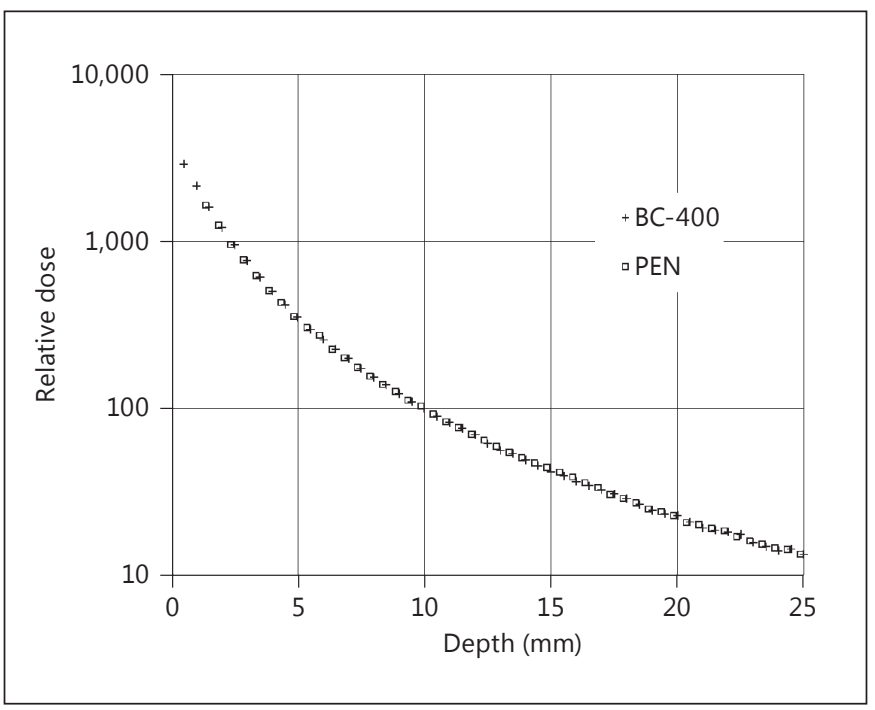

Fig. 7. Comparison between measurements of a ${ }^{125} \mathrm{I}$ eye applicator with a PEN and a calibrated PVT scintillator type BC-400. Note that the initial profile gradient is larger than for COMS (Collaborative Ocular Melanoma Study) plaques, due to the position of the 8 seeds close to the applicator centre. The size of the markers represents the measuring uncertainty $(\mathrm{k}=1)$. The dose rate at the normalization point at $10 \mathrm{~mm}$ was $15 \mathrm{mGy} / \mathrm{min}$.

\section{Discussion}

Our investigations clearly demonstrated that a PEN scintillator is principally applicable as a clinical dosimeter for a wide range of clinically used photon and electron beam qualities, with different energies and dose rates, ranging from $<0.1 \mathrm{mGy} / \mathrm{min}$ to several $\mathrm{Gy} / \mathrm{min}$. This applicability encompasses especially the still difficult field of ophthalmic plaque dosimetry. In all cases, the response of a PEN detector was in excellent agreement to the signal of reference detectors. The applicability is comparable or superior to other types of detectors with similar application fields, such as PVT scintillators, small diodes or Gafchromic EBT-3 film.

The dependence of the electron and photon cross sections upon the particle energy shows a similar behaviour for PEN and water. Thus, PEN can be regarded as dosimetrically almost water equivalent. The material is cheaper than conventional scintillators and easy to manufacture into different geometric designs for special dosimetric tasks. It remains resistant against chemical and physical influences during the manufacturing process, especially thermal stress, and does not emit toxic substances. The light output of every specimen was found

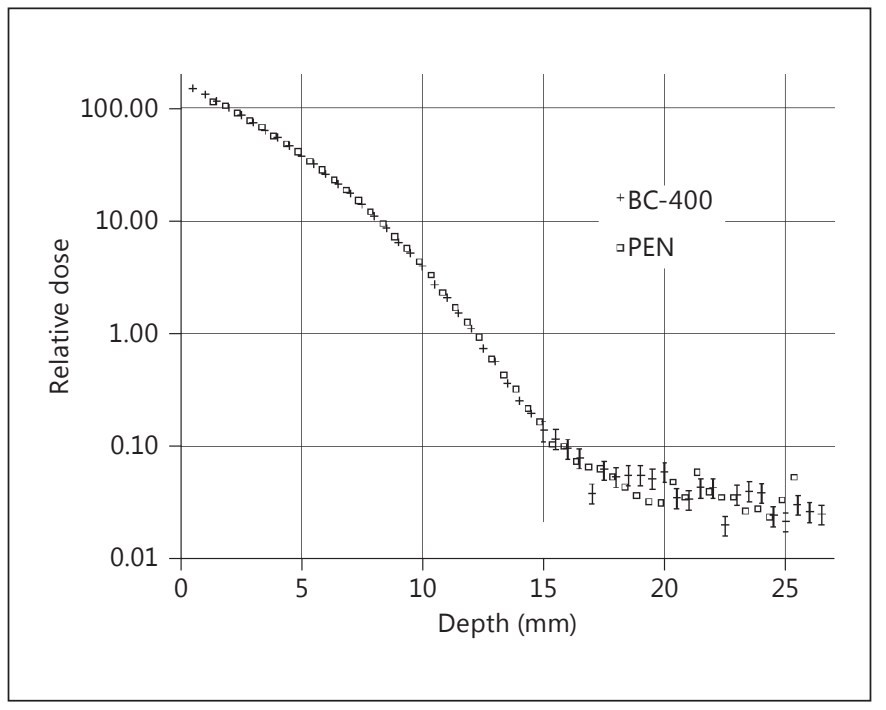

Fig. 8. Comparison between measurements of a ${ }^{106} \mathrm{Ru} /{ }^{106} \mathrm{Rh}$ eye applicator with a PEN and a calibrated PVT scintillator type BC400. The size of the markers represents the measuring uncertainty for the $\beta$ profile $(k=1)$. In the region of the photon background, error bars are shown only for the PVT measurements, for clarity. In fact, the uncertainty is the same for both detectors (20\%). The dose rate at the reference point at $2 \mathrm{~mm}$, according to [23], was 88 $\mathrm{mGy} / \mathrm{min}$.

to be comparable to that of a PVT scintillator of the same size.

In the problematic field of photon energies below 40 $\mathrm{keV}$, such as for ${ }^{125} \mathrm{I}$ eye plaque dosimetry, PEN demonstrates notable advantages compared to PVT. The light responses of both PVT and PEN scintillators to the dose rate in this energy range are linear but reduced compared to the values obtained for $\beta$ radiation $\left({ }^{106} \mathrm{Ru} /{ }^{106} \mathrm{Rh}\right.$ applicator) or photon radiation with higher energies. For PEN, however, this disadvantageous reduction was found to be considerably lower than for PVT.

For PVT, we determined a reduction factor of approximately 3.26 in earlier investigations [22], in accordance with other researchers $[36,37]$. We found this factor to be depending on the combined influence of two effects: the ratio of the mass absorption coefficients of PVT and water (fig. 1), inducing a reduction by a factor of approximately 2.5 , and an additional decrease of the light response caused by the so-called quenching effect, i.e. the deposition of radiation energy in the scintillator without resulting light emission.

Taking into account only the mass absorption coefficients of the two materials, we expected a response for PEN reaching about $150 \%$ of the PVT value but found a 
much larger result of approximately $190 \%$. This quantitative difference between the expected and measured values can be fully explained by the quenching effect in PVT. The ratio for PEN is in quite good agreement with the value expected from the ratio of the mass absorption coefficients of this material and water. Thus, our recent investigations indicate that there is no or only a small quenching effect in PEN. This absence of a distinct quenching effect for low-energy photons makes PEN also an interesting material for further investigations as a detector, for instance for the dosimetry of therapeutic proton beams $[38,39]$.

\section{Conclusion}

PEN was recognized as a novel plastic scintillator material in 2011. Due to its density and atomic composition, it features an approximate dosimetric water equivalence and provides fine properties for the dosimetry of both electron and photon radiation. It allows for a minimal disturbance of the radiation field during the measuring process, especially in dosimetrically difficult situations, such as the dosimetry of small radiation fields and radio- active eye plaques. This material primarily emits light with a similar wavelength and gain as conventional PVTbased plastic scintillators with wavelength shifters. Due to its superior mechanical, chemical and physical characteristics, however, PEN is much easier to process and manufacture as a detector than PVT, especially when detector designs for special measuring situations are required. In our investigations, PEN scintillators proved to be suitable detectors for a wide range of applications in the dosimetry of clinical photon and electron fields, including low-energy photon radiation from ${ }^{125} \mathrm{I}(25-35 \mathrm{keV})$ and ${ }^{192} \mathrm{Ir}$ sources (approximately $300-600 \mathrm{keV}$ ), ${ }^{106} \mathrm{Ru} /{ }^{106} \mathrm{Rh} \beta$ radiation (3.5 MeV maximal energy) and photon and electron beams from a linear accelerator (from 6 to $18 \mathrm{MeV}$ ). Furthermore, PEN features considerably better properties than PVT in case of the dosimetry of low-energy photon radiation, such as from ${ }^{125} \mathrm{I}$ eye plaques. Hence, we recommend PEN as an ideal material for the dosimetry of ophthalmic plaques.

\section{Statement of Ethics}

The described dosimetric research did not include studies on humans or animals.

\section{References}

1 Nag S, Quivey JM, Earle JD, Followill D, Fontanesi J, Finger PT, American Brachytherapy Society: The American Brachytherapy Society recommendations for brachytherapy of uveal melanomas. Int J Radiat Oncol Biol Phys 2003;56:544-555.

2 American Brachytherapy Society - Ophthalmic Oncology Task Force; ABS - OOTF Committee: The American Brachytherapy Society consensus guidelines for plaque brachytherapy of uveal melanoma and retinoblastoma. Brachytherapy 2014;13:1-14.

3 Reich H, Harder D: Die Sondenmethode für Photonen- und Elektronenstrahlung; in Reich H (ed): Dosimetrie ionisierender Strahlung: Grundlagen und Anwendungen. Stuttgart, BG Teubner, 1990, pp 206-221.

4 Beddar AS, Mackie TR, Attix FH: Waterequivalent plastic scintillation detectors for high-energy beam dosimetry: I. Physical characteristics and theoretical consideration. Phys Med Biol 1992;37:1883-1900.

5 Beddar AS, Mackie TR, Attix FH: Waterequivalent plastic scintillation detectors for high-energy beam dosimetry: II. Properties and measurements. Phys Med Biol 1992;37: 1901-1913.
6 Flühs D, Heintz M, Indenkämpen F, Wieczorek C: Direct reading measurement of absorbed dose with plastic scintillators - the general concept and applications to ophthalmic plaque dosimetry. Med Phys 1996;23: 427-304.

7 Beddar AS: Water equivalent plastic scintillation detectors in radiation therapy. Radiat Prot Dosimetry 2006;120:1-6.

8 Perera $\mathrm{H}$, Williamson JF, Monthofer SP, Binns WR, Klarmann J, Fuller GL, Wong JW: Rapid two-dimensional dose measurement in brachytherapy using plastic scintillator sheet: linearity, signal-to-noise ratio, and energy response characteristics. Int J Radiat Oncol Biol Phys 1992;23:1059-1069.

9 Geso M, Robinson N, Schumer W, Williams $\mathrm{K}$ : Use of water-equivalent plastic scintillator for intravascular brachytherapy dosimetry. Australas Phys Eng Sci Med 2004;27:5-10.

10 Lambert J, McKenzie DR, Law S, Elsey J, Suchowerska N: A plastic scintillation dosimeter for high dose rate brachytherapy. Phys Med Biol 2006;51:5505-5516.

11 Guillot M, Beaulieu L, Archambault L, Beddar $S$, Gingras L: A new water-equivalent 2D plastic scintillation detectors array for the dosim- etry of megavoltage energy photon beams in radiation therapy. Med Phys 2011;38:67636774.

12 Guillot M, Gingras L, Archambault L, Beddar S, Beaulieu L: Spectral method for the correction of the Cerenkov light effect in plastic scintillation detectors: a comparison study of calibration procedures and validation in Cerenkov light-dominated situations. Med Phys 2011;38:2140-2150.

13 Therriault-Proulx F, Beddar S, Briere TM, Archambault L, Beaulieu L: Technical note: removing the stem effect when performing Ir-192 HDR brachytherapy in vivo dosimetry using plastic scintillation detectors: a relevant and necessary step. Med Phys 2011;38:2176-2179.

14 Therriault-Proulx F, Briere TM, Mourtada F, Aubin S, Beddar S, Beaulieu L: A phantom study of an in vivo dosimetry system using plastic scintillation detectors for real-time verification of 192Ir HDR brachytherapy. Med Phys 2011;38:2542-2551.

15 Archambault L, Briere TM, Ponisch F, Beaulieu L, Kuban DA, Lee A, Beddar S: Toward a real-time in vivo dosimetry system using plastic scintillation detectors. Int J Radiat Oncol Biol Phys 2010;78:280-287. 
16 Beierholm AR, Behrens CF, Andersen CE: Dosimetric characterization of the Exradin W1 plastic scintillator detector through comparison with an in-house developed scintillator system. Radiat Meas 2014;69:50-56.

17 Buranurak S, Andersen CE, Beierholm AR, Lindvold LR: Temperature variations as a source of uncertainty in medical fiber-coupled organic plastic scintillator dosimetry. Radiat Meas 2013;56:307-311.

18 Lindvold LR, Beierholm AR, Andersen CE: Fiber-coupled organic plastic scintillator for on-line dose rate monitoring in $6 \mathrm{MV} \mathrm{X}$-ray beam for external radiotherapy. Proc SPIE 7559, Optical Fibers and Sensors for Medical Diagnostics and Treatment Applications X 2010;7559.

19 Eichmann M, Flühs D, Spaan B: Development of a high precision dosimetry system for the measurement of surface dose rate distribution for eye applicators. Med Phys 2009;36:46344643.

20 Flühs D, Bambynek M, Heintz M, Indenkämpen F, Kolanoski H, Wegener D, Sauerwein W, Quast U: Dosimetry and design of radioactive eye plaques. Front Radiat Ther Oncol 1997;30:26-38.

21 Eichmann M, Krause T, Flühs D, Spaan B: Development of a high-precision xyz-measuring table for the determination of the $3 \mathrm{D}$ dose rate distributions of brachytherapy sources. Phys Med Biol 2012;57:N421-N429.

22 Flühs D, Anastassiou G, Wening J, Sauerwein $\mathrm{W}$, Bornfeld N: The design and the dosimetry of bi-nuclide radioactive ophthalmic applicators. Med Phys 2004;31:1481-1488.
23 ISO 21439:2009, Clinical Dosimetry - Beta Radiation Sources for Brachytherapy. 2009.

24 Archambault L, Beddar AS, Gingras L, Roy R, Beaulieu L: Measurement accuracy and Cerenkov removal for high performance, high spatial resolution scintillation dosimetry. Med Phys 2006;33:128-135.

25 Lambert J, Yin Y, McKenzie DR, Law S, Suchowerska N: Cerenkov-free scintillation dosimetry in external beam radiotherapy with an air core light guide. Phys Med Biol 2008;53: 3071-3080.

26 Liu PZ, Suchowerska N, Lambert J, Abolfathi P, McKenzie DR: Plastic scintillation dosimetry: comparison of three solutions for the $\mathrm{Ce}$ renkov challenge. Phys Med Biol 2011;56: 5805-5821.

27 Yoo WJ, Shin SH, Jeon D, Hong S, Kim SG, Sim HI, Jang KW, Cho S, Lee B: Simultaneous measurements of pure scintillation and $\mathrm{Ce}$ renkov signals in an integrated fiber-optic dosimeter for electron beam therapy dosimetry. Opt Express 2013;21:27770-27779.

28 Archambault L, Beaulieu L, Beddar SA: Comment on 'Plastic scintillation dosimetry: comparison of three solutions for the Cerenkov challenge'. Phys Med Biol 2012;57:36613665; discussion 3667-3673.

29 Beddar AS, Suchowerska N, Law SH: Plastic scintillation dosimetry for radiation therapy: minimizing capture of Cerenkov radiation noise. Phys Med Biol 2004;49:783-790.

30 Saint-Gobain Crystals Organic Scintillation Materials, 2014. http://www.crystals.saintgobain.com/uploadedFiles/SG-Crystals/ Documents/SGC\%20Organics\%20Brochure. pdf.
31 Nakamura H, Shirakawa Y, Takahashi S, Shimizu H: Evidence of deep-blue photon emission at high efficiency by common plastic. EPL 2011;95:22001.

32 NIST Physical Reference Data - Radiation Dosimetry Data, 2014. http://physics.nist. gov/PhysRefData/Star/Text/ESTAR-u.html.

33 NIST Physical Reference Data - X-Ray and Gamma-Ray Data, 2014. http://www.nist. gov/pml/data/xcom/index.cfm.

34 DIN 6800-1: Procedures of dosimetry with probe-type detectors for photon and electron radiation - part 1: general. 2013.

35 DIN 6800-2: Procedures of dosimetry with probe-type detectors for photon and electron radiation - part 2: ionization chamber dosimetry of high energy photon and electron radiation. 2008

36 Williamson JF, Dempsey JF, Kirov AS, Monroe JI, Binns WR, Hedtjarn H: Plastic scintillator response to low-energy photons. Phys Med Biol 1999;44:857-871.

37 Peralta L, Rego F: Response of plastic scintillators to low-energy photons. Phys Med Biol 2014;59:4621-4633.

38 Wang LL, Perles LA, Archambault L, Sahoo N, Mirkovic D, Beddar S: Determination of the quenching correction factors for plastic scintillation detectors in therapeutic high-energy proton beams. Phys Med Biol 2012;57: 7767-7781.

39 Ghergherehchi M, Afarideh H, Ghannadi M, Mohammadzadeh A, Aslani GR, Boghrati B: Proton beam dosimetry: a comparison between a plastic scintillator, ionization chamber and Faraday cup. J Radiat Res 2010;51: 423-430. 